J. Anim. Ecol. 42, 521-530, October 1973

\title{
A RE-INTERPRETATION OF GAUSE'S POPULATION EXPERI- MENTS BY MEANS OF SIMULATION
}

BY J. GOUDRIAAN AND C. T. DE WIT

Department of Theoretical Production Ecology, Agricultural University, Wageningen, The Netherlands

BLACKWELL SCIENTIFIC PUBLICATIONS OXFORD LONDON EDINBURGH MELBOURNE 


\title{
A RE-INTERPRETATION OF GAUSE'S POPULATION EXPERI- MENTS BY MEANS OF SIMULATION*
}

\author{
By J. GOUDRIAAN AND C. T. DE WIT
}

Department of Theoretical Production Ecology, Agricultural University, Wageningen, The Netherlands

\section{INTRODUCTION}

Lotka (1925) and Volterra (1928) described the growth of two interacting species by the differential equations:

$$
\begin{aligned}
& \frac{d S_{1}}{d t}=R_{1} \times S_{1} \times\left(1-\frac{S_{1}}{K_{1}}-\alpha \frac{S_{2}}{K_{2}}\right) ; \\
& \frac{d S_{2}}{d t}=R_{2} \times S_{2} \times\left(1-\beta \frac{S_{1}}{K_{1}}-\frac{S_{2}}{K_{2}}\right) .
\end{aligned}
$$

The parameters $K$ are the maximum size of the population that can be sustained in the particular environment and the constants $R$ the relative rate of increase of the species when the population is still small compared with the value of $K$. The parameters $\alpha$ and $\beta$ characterize the interference of the species.

Gause (1934) treated several series of experiments with simple organisms in his wellknown book The Struggle for Existence to evaluate experimentally the above differential equations. However, it appeared that, even in very simple situations, the behaviour of the organisms was more complicated than assumed by the mathematical expressions.

In such situations, two courses of action may be pursued. The first is to design additional elementary experiments. The second is to use an appropriate technique of simulation to describe and quantify the system. This allows the introduction of much more realistic inter-relations and boundary conditions than the classical mathematical analyses.

Simulation of ecological systems will be illustrated by re-interpreting some of the still elegant experiments of Gause on competition between yeast and Paramecium species. The simulation itself is done by use of a Continuous System Modelling Program (IBM; CSMP/360 Manual). The use of this particular programming technique in ecology has been explained elsewhere (Brennan et al. 1970; de Wit \& Goudriaan 1973), so that the actual simulation programs are not given here. Listings are, however, available on request.

\section{COMPETITION BETWEEN YEAST SPECIES}

Gause cultivated the yeasts Saccharomyces and Schizosaccharomyces in mono- and in mixed culture. They were grown in test tubes and their growth was determined by volumetric measurements. The yeast was fed on sugar, producing $\mathrm{CO}_{2}$ and ethanol (alcohol) during the process. There was an excess of sugar available, and the limiting factor for

* Paper read at the British Ecological Society's Symposium on Mathematical Models in Ecology, March 1971. 
growth was the presence of alcohol. As mentioned by Gause (1934) alcohol in the medium may kill the young buds emerging from the yeast and inhibit further growth.

The experimental results are presented in Fig. 1. Both species exhibit a more or less sigmoid growth in monoculture. The growth process is described as follows.

The amount of yeast is the integral of its growth rate which is equal to the product of the relative growth rate without alcohol, the amount of yeast and a factor which represents the growth-reducing effect of alcohol. It is assumed that the reduction is proportional to the alcohol concentration. When the alcohol concentration reaches the specified maximum, growth is finished. The rate of increase in alcohol concentration, which must be also integrated, is obtained by multiplying the growth rate of the yeast and an alcohol production factor. These statements describe all structural relations. The data for the yeast Saccharomyces are obtained by visually matching the simulated curve to the
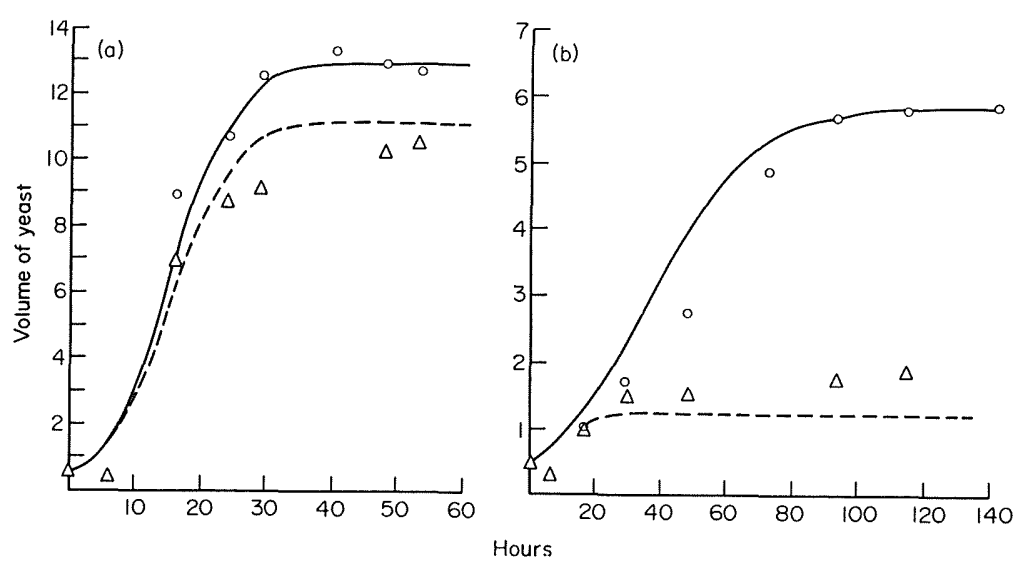

FIG. 1. The growth of yeast in monoculture and in mixture: (a) Saccharomyces; (b) Schizosaccharomyces. The simulated curves for the mixtures are independent of the associated experimental data. $O$, Measured monoculture; $\Delta$, measured mixed culture; - , simulated monoculture - - , simulated mixed culture.

observations of the monoculture. The simulation has to proceed for $100 \mathrm{~h}$ of experimental time and output is wanted every hour. The unit of time is the same as for the relative growth rate. Graphical and printed output is requested for the amount of yeast and printed output only for the alcohol percentage.

With the above information in its proper format, CSMP writes a FORTRAN program for numerical integration which is executed, in this case by the method of Runge-Kutta, whereby the size of the time intervals is adapted to the rate of change of the contents of the integral.

The linear relation between the alcohol concentration and the reduction factor may be replaced by any curvilinear expression; however, the scatter of the observation is so large in this case that there is no reason to deviate from a linear relation.

For the mixed culture, it is assumed that the two species interfere only by the production of alcohol. This means that the parameter values are the same as in the monocultures.

The results of the simulation and the experimental data are given in Fig. 1. The simulated results for the mixture agree reasonably for the yeast Schizosaccaromyces, but the actual growth of the yeast Saccharomyces is slower than the simulated growth. This 
might imply that the situation is complicated by the influence of another waste product, e.g. another alcohol, besides ethanol. Introduction of this new substance into the simulation program without more experimental data will not yield more relevant information.

\section{COMPETITION BETWEEN PARAMECIUM SPECIES \\ Description of the experiment}

In another experiment, Gause cultivated the Paramecium species caudatum and aurelia in mono- and in mixed culture. They were grown in test tubes with $5 \mathrm{~cm}^{3}$ of Oosterhout's balanced physiological solution buffered at $\mathrm{pH} 8 \cdot 0$. Every day, the medium was centrifuged and the Protozoa put into a fresh solution to prevent accumulation of waste products. At the same time, a standardized amount of Bacillus pyocyaneus was given as daily food. Before being placed in the centrifuge the medium was carefully stirred, and $0.5 \mathrm{~cm}^{3}$, or one-tenth of the liquid, was taken out and the number of infusoria in it counted. Two series were made, one with provision of 'one loop' of bacteria each day and the other with a 'half loop' of bacteria.
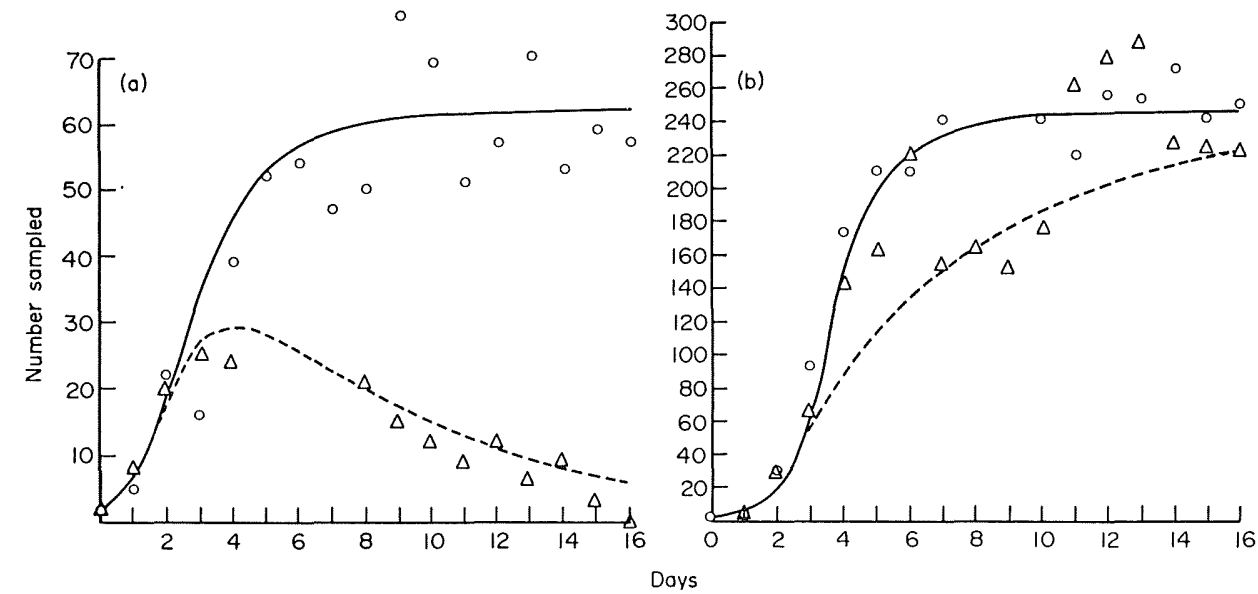

FIG. 2. The growth of Paramecium in monoculture and in mixture in the 'one-loop' experiment: (a) P. aurelia; (b) $P$. caudatum. $O$, Measured monoculture; $\triangle$, measured mixed culture; - , simulated monoculture; --- , simulated mixed culture.

The number of Protozoa counted in each treatment is presented in Fig. 2 as a function of time. The growth of the monoculture follows a sigmoid course as for the yeast. Here, the termination of growth is not due to accumulation of waste products but to the exhaustion of the daily food supply. In due course the amount of food is just sufficient to maintain the Protozoa and to provide enough food to replace the one-tenth that is removed by sampling each day.

In mixed culture, both species at first increase in number but later one species reaches a maximum and then declines, whereas the other species continues to increase until the first has vanished.

\section{Assumptions on growth and death}

Growth is caused by consumption of food and is linearly related to it. There is a 
constant ratio between the consumed food and the newly grown amount of Paramecium: the conversion factor is expressed in animals per loop. It is supposed that a relative death rate exists, which is independent of the density. In addition to this natural death, onetenth of the population is removed every day, both species suffering equally. The rate of food consumption, which determines the growth rate, is supposed to be proportional to the number of Protozoa, to the concentration of food and to the rate of searching the water for bacteria. The rate of consumption is limited by a maximum rate of digestion of the animal. The above assumptions are quantitatively formulated.

\section{The simulation program}

The amount of Protozoa of each species is an integral with an initial amount and net growth rate. The net growth rate is the gross growth rate by food consumption minus the death rate and minus the sampled amount. The gross growth rate is the consumption of food times the conversion factor. The consumption is the amount of Protozoa times the consumption per animal, which is the rate of searching water times the concentration of food. There is an upper rate of digestion. The natural death is the relative death rate times the amount of Protozoa.

The sampling is zero, except once a day when it is one-tenth of the amount of Protozoa. The food is another integral, which decreases by the food consumption by both species.

Once a day, the food is replaced to the initial level $L$ which is either 0.5 (half-loop experiment) or 1.0 (one-loop experiment) loop per total volume.

As a discontinuity is introduced each day by the sampling and the food addition, it is necessary to integrate by the rectilinear method of Euler. For comparison with Gause's data, it suffices to print the size of the samples each day, but a more frequent printing of the population numbers is necessary for studying the behaviour of the simulated populations. Unlike the one for yeast, this simulation program cannot be represented by a set of simple differential equations.

\section{Derivation of the values of the parameters}

Gause observed that the medium became rapidly transparent at the end of the experiment. At that stage the food $(L)$ is consumed at a high rate and very quickly depleted. The course of the growth of the population is then hardly affected by the rate of searching water (RSW) or the maximum digestion rate (MRDIG). The size of, and the rate of approach to the equilibrium population are therefore mainly dependent on the food conversion factor (CONVF) and the relative death rate (RDR). Some approximate calculations suffice to make first estimates of these two parameters that are subsequently improved by simulation. At first, it is noted that the gross daily growth depends only on the conversion factor and the total daily food and is about $L \times$ CONVF. The daily death depends on the size of the population $(H)$ and is roughly given by $H \times(\mathrm{RDR}+0 \cdot 1)$. This is a negative feedback, so that the time constant governing the approach to the equilibrium population is about $1 /(\mathrm{RDR}+0 \cdot 1)$. Gause's experiments suggest a value of 2 days for either species, resulting in a RDR of 0.4 day $^{-1}$. Simulation shows that $0.45 \mathrm{day}^{-1}$ for both species is a more acceptable value. At equilibrium, $L \times \mathrm{CONVF}=\mathrm{H} \times(\mathrm{RDR}+0 \cdot 1)$, so that the conversion factor can also be determined. For $P$. aurelia, the equilibrium population is about 4900 individuals in $5 \mathrm{~cm}^{3}$ with one loop of bacteria per day, so that CONVF is about 2700 P. aurelia per loop. Simulation shows that 3000 is a more accurate value. The equilibrium population of $P$. caudatum is four times smaller so that CONVF is 750 P. caudatum per loop. 
RSW and MRDIG are now estimated from the data for the first days when the food is not depleted. Assuming that the consumption per individual is limited by the maximum rate of digestion, exponential growth will occur with a relative growth rate of about MRDIG $\times$ CONVF $-\mathrm{RDR}-0 \cdot 1$. The relative growth rate of $P$. aurelia is about $1 \cdot 5 \mathrm{day}^{-1}$ during the first days, so that the maximum rate of digestion (MRDIG) will be in the order of 0.001 loop day ${ }^{-1}$ aurelia $^{-1}$.

The rate of searching the water (RSW) manifests itself when the concentration of the bacteria is below the saturation level of the animal. As the relative growth rate of the 0.5 and the one-loop treatments do not differ very much, this saturation level may be estimated at 0.5 loop, so that a first estimate for RSW is 0.002 of the volume of $5 \mathrm{~cm}^{3}$ per Paramecium per day.

At this stage of growth the interactions are very difficult to analyse, so that these estimates may be a factor of 5-10 different from the actual values that are found by simulation. But even very rough estimates reduce considerably the number of simulations that are necessary to arrive at acceptable values of the parameters.

Repeated simulation runs and comparison with the experimental data give the following as optimal values.

Relative death rate (RDR)

Conversion factor (CONVF)

Rate of digestion (MRDIG)

Rate of searching water (RSW)

$$
\begin{aligned}
& \text { P. aurelia P. caudatum Units } \\
& \begin{array}{ccl}
0.45 & 0.45 & \text { Day }^{-1} \\
3000 & 750 & \text { Animal loop }^{-1}
\end{array} \\
& 0.5610^{-3} \quad 2 \cdot 2410^{-3} \text { Loop animal }^{-1} \text { day }^{-1} \\
& 0.006 \quad 0.006 \quad V^{0} \quad \text { animal }^{-1} \text { day }^{-1}
\end{aligned}
$$

By volume is meant the total volume of $5 \mathrm{~cm}^{3}$. The relative death rate and the rate of searching water turn out to have the same value for both species.

The results of simulation runs with these values are presented in Fig. 2. The smooth curve is obtained by connecting the simulated samples. The population itself varies considerably during the day as shown in Fig. 3 for two extreme situations.

\section{Introduction of stochastic phenomena}

Gause's observations have a large scatter which will now be considered. Two phenomena are accessible for computation, the sampling and the death process. Up to this point, we assumed that Gause was able to separate exactly one-tenth of the population and count it. Similarly, we supposed that the death process is continuous and determined exactly by the relative death rate. However, the number of deaths during each time interval is better described by a Poisson distribution with expectation $\mathrm{RDR} \times \mathrm{H} \times \mathrm{DELT}$ where DELT is the time interval of simulation. In a similar way the sampling process may be presented by drawing the sample once a day from a Poisson distribution with one-tenth of the population as expected value.

In Fig. 4 three runs are represented, one with a fully deterministic program, one with sampling deterministic and dying stochastic, and one with both processes stochastic. The simulated size of the sample is represented, rather than the whole population. Visually, the agreement between the observed and simulated scatter is good, in the run where the sampling technique is introduced as a source of scatter. The variance for $P$. aurelia is considerably smaller than that of $P$. caudatum, because the population is four times larger. However, it is striking that the scatter due to sampling is far greater than that due to the stochastic death process, although about $45 \%$ of the population dies each day and only $10 \%$ is sampled. A simple calculation can explain this. Let the equilibrium 
population consist of 1000 individuals. The average sample size is 100 , so that the standard deviation is $\sqrt{100}=10$ individuals. Each day an average of 450 individuals die, the standard error being about $\sqrt{0 \cdot 45 \times 0 \cdot 55 \times 1000}=16$. Because one-tenth is sampled, this gives
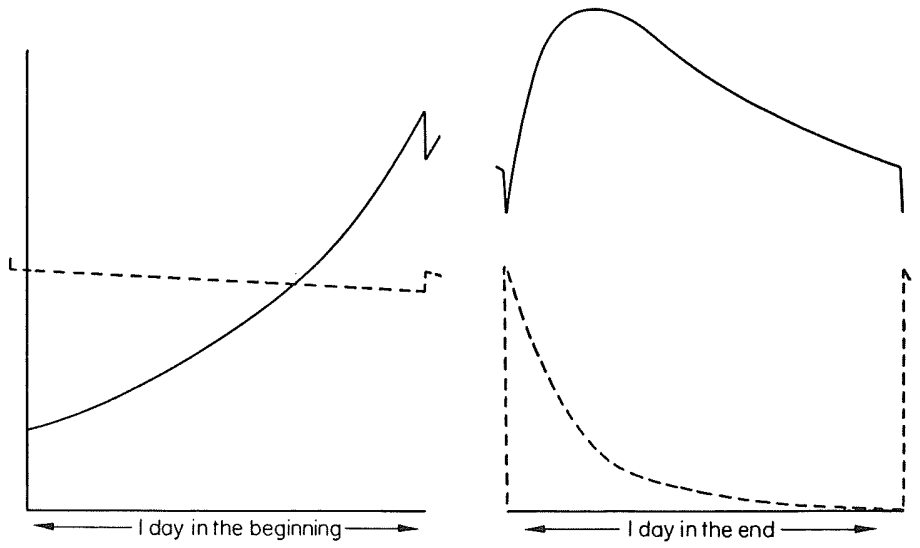

FIG. 3. The daily course of population and food at the beginning and the end of the experiment. - - Population; - - - food (arbitrary scales).

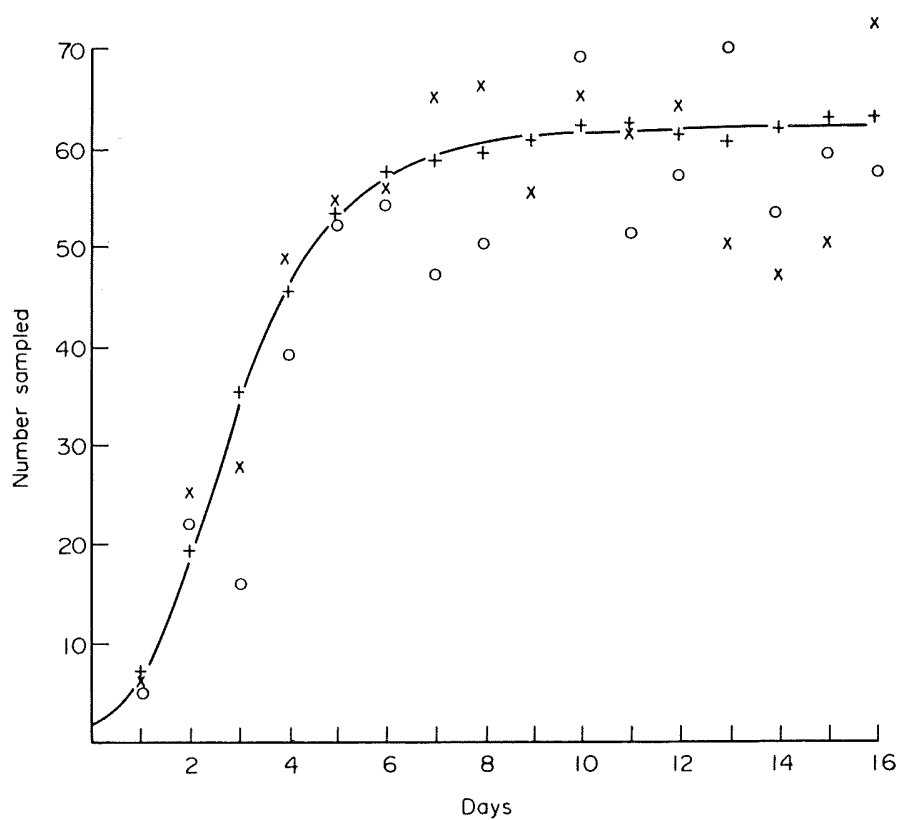

FIG. 4. A comparison of the results of deterministic and stochastic simulations with the experimental result for Paramecium caudatum in monoculture. $\bigcirc$, Measured sample; + , deterministic sample, but stochastic death process; $\times$, both sampling and death processes stochastic; —_, deterministic simulation.

a standard deviation in the sample of about 1.6 and this is only one-sixth of the standard deviation caused by the sampling. Because the death process is distributed over the day, some deviations are levelled by the negative feed-back throughout the day. This again diminishes the influence on the scatter of the death process. 


\section{DISCUSSION}

The values of the parameters for the Paramecium model were estimated by visually fitting.

Admittedly there are better methods, but the scatter of the observations is so large that these do not provide a much sharper criterion. Ross (1972) used a density-dependent birth-and-death process, restricted to the logistic equations. He estimated the parameters by means of a $\chi^{2}$ method. We showed here that a stochastic birth-and-death process, like he used, cannot explain the scatter, but that the sampling technique is the main cause.

In Fig. 3 the simulated course of population and food is represented during a day at the beginning and at the end of the experiment. At the end, the food is rapidly consumed during the first hours of the day. Both species suffer here equally from sampling and natural death, but have a different ability to consume newly given food. This is the reason that one species replaces the other as soon as all food is exhausted. Gause's explanation that the replacement is caused by the different rates of filling the gap caused by sampling is in the right direction, but is inadequate. An additional cause of disappearance must be introduced to explain the time constant of about 2 days near the equilibrium situation. Sampling alone would give rise to a time constant of about 10 days.

Gause analysed and described the results of the experiment with both yeast and Paramecium by means of the Lotka-Volterra equations given in the Introduction. As far as the yeast is concerned, the simulation program is equivalent to the differential equations with the maximum amount of yeast $K$ equal to the quotient of the maximum alcohol concentration and the alcohol production factor. The only difference is that it is assumed implicity in the differential equations that the initial amount of alcohol equals the initial amount of yeast times the alcohol production factor, whereas the initial amount of alcohol in the experiment and in the simulation program is zero.

The attempt of Gause to describe the experiments with Protozoa by means of the Lotka-Volterra equations was not very successful. For symmetry of the sigmoid curves, the time constant for the approach to the equilibrium population must be equal to the inverse of the relative growth rate in the early stage. He found that the growth curves of the Paramecium in monoculture were not sigmoid, which forced him to change the parameters in the course of growth. The present model does not require symmetrical sigmoid growth curves and in this respect it is in better agreement with the data than models based on the Lotka-Volterra equations.

Leslie (1957) reinterpreted Gause's data also on the basis of the logistic equation which he presented in the following form:

$$
\frac{d S_{1}}{d t}=S \times(\mathrm{RGR}-\alpha \times S) .
$$

The two values RGR and $\alpha$ are calculated from the best fitting curve. $R G R / \alpha$ is the maximum number and $R G R$ is the relative growth rate.

In the mixed population, the extension of this equation is

$$
\frac{d S_{1}}{d t}=S_{1} \times\left(\mathrm{RGR}_{1}-\alpha_{1} \times S_{1}-\beta_{1} \times S_{2}\right) ;
$$




$$
\frac{d S_{2}}{d t}=S_{2} \times\left(\mathrm{RGR}_{2}-\alpha_{2} \times S_{1}-\beta_{2} \times S_{2}\right) .
$$

Leslie assumed that the alphas and betas for both species were the same on the strength of the following argument: 'Now in the very simplified experimental conditions in which these two closely related species of Paramecium were living, we might assume that the magnitude of the effect which $P$. aurelia had on the relative rate of increase of $P$. caudatum was much the same as that which it had on its own relative rate of increase'. However, the effect of this argument depends largely on the way of writing Eqn 3. If written as in the introduction.

$$
\begin{aligned}
& \frac{d S_{1}}{d t}=S_{1} \times \mathrm{RGR}_{1} \times\left(1-\frac{\alpha_{1} \times S_{1}}{\mathrm{RGR}_{1}}-\frac{\beta_{1} \times S_{2}}{\mathrm{RGR}_{1}}\right) \\
& \frac{d S_{2}}{d t}=S_{2} \times \mathrm{RGR}_{2} \times\left(1-\frac{\alpha_{2} \times S_{1}}{\mathrm{RGR}_{2}}-\frac{\beta_{2} \times S_{2}}{\mathrm{RGR}_{2}}\right)
\end{aligned}
$$

then the alphas and the betas for both species would not be the same on the strength of the above argument. Equal mutual effects in this way of writing would lead to a situation where both species survive, as for the yeast.

Another argument can be conceived to support the assumption that $\alpha_{1}=\alpha_{2}$ and $\beta_{1}=\beta_{2}$. Let it be assumed that there is a constant flow of food. In monoculture, where the whole flow is available for one species the maximum number of $S_{1}$ is $K_{1}$. In the mixed culture, the flow is divided into two parts. If the velocity of eating is proportional to the velocity of growing, the ratio of the available flows of food is $\mathrm{RGR}_{1} \times S_{1}$ over $\mathrm{RGR}_{2} \times S_{2}$. The resulting maximum of $S_{1}$ is then:

$$
\mathrm{K}_{1}^{\prime}=\frac{\mathrm{RGR}_{1} \times S_{1}}{\mathrm{RGR}_{1} \times \mathrm{S}_{1}+\mathrm{RGR}_{2} \times \mathrm{S}_{2}} \times \mathrm{K}_{1}
$$

and likewise for species $S_{2}$.

Substitution in

$$
\frac{d S_{1}}{d t}=\mathrm{RGR}_{1} \times S_{1} \times\left(1-\frac{S_{1}}{K_{1}^{\prime}}\right)
$$

gives

$$
\frac{d S_{1}}{d t}=\mathrm{RGR}_{1} \times \mathrm{S}_{1} \times\left(1-\frac{S_{1}}{K_{1}}-\frac{\mathrm{RGR}_{2}}{\mathrm{RGR}_{1}} \times \frac{S_{2}}{K_{1}}\right) .
$$

Bringing RGR back between the brackets, as done by Leslie, gives:

$$
\frac{d S_{1}}{d t}=S_{1} \times\left(\mathrm{RGR}_{1}-\frac{\mathrm{RGR}_{1} \times S_{1}}{K_{1}}-\frac{\mathrm{RGR}_{2} \times S_{2}}{K_{1}}\right) ;
$$

and, for $S_{2}$,

$$
\frac{d S_{2}}{d t}=S_{2} \times\left(\mathrm{RGR}_{2}-\frac{\mathrm{RGR}_{1} \times S_{1}}{K_{2}}-\frac{\mathrm{RGR}_{2} \times \mathrm{S}_{2}}{K_{2}}\right) .
$$

Making the assumption that $K_{1}$ equals $K_{2}$ when expressed in dry weight rather than in numbers it can be seen that $\mathrm{RGR}_{1} / K_{1}=\mathrm{RGR}_{1} / K_{2}$ and that $\mathrm{RGR}_{2} / K_{1}=\mathrm{RGR}_{2} / K_{2}$. 
This corresponds with Leslie's assumption that $\alpha_{1}=\alpha_{2}$ and $\beta_{1}=\beta_{2}$. Of course, $S_{1}$ and $S_{2}$ should be expressed in dry weight in this situation. For the derivation of Eqn 8, we have made more assumptions than for the more detailed analysis by the previous simulation, but as a first rough description it is acceptable.

De Wit (1960) also dealt with the Paramecia and applied an integrated form of the logistic equation

$$
\begin{aligned}
& \left.O_{a}(t+2)=\beta_{1}\left(O_{a}(t) / \beta_{1} O_{a}(t)+\beta_{2} O_{c}(t)+1\right)\right) \times \Omega_{a} ; \\
& \left.O_{c}(t+2)=\beta_{2}\left(O_{c}(t) / \beta_{1} O_{a}(t)+\beta_{2} O_{c}(t)+1\right)\right) \times \Omega_{c} .
\end{aligned}
$$

The results of these iterative equations are much the same as those of Eqn 4. The underlying philosophy, however, is different. De Wit deals with the culture of the Paramecia in a way analogous to his conception about the competition between plant species in a crop. The spaces occupied per individual after 2 days, when sown at a very low density, is equal to $\beta$. By means of the process of repeated harvesting and sowing, the replacement is accomplished. The values of $\beta$ and $\Omega$ may be derived from the values of RGR and $K$ and depend also on the integration period.

The present treatment of the experiments by means of simulation techniques shows clearly that, in this way, a better understanding of the ecological systems is obtained than by means of a classical mathematical analysis. Sophisticated mathematical techniques for the solution of differential equations like Laplace transformations and series development can also be replaced, to a large extent, by simulation. However, this does not mean that simulation programs can be written and used without knowledge of mathematical principles. This appears in this example by the use of mathematical relations to reduce the trial and error curve-fitting procedure.

It can also be concluded that a good analysis or even a simple system is only possible with a good understanding of the relevant physiology of the organisms involved and of the details of the experimental situation.

\section{SUMMARY}

(1) Gause's population experiments on yeast and Paramecia have been re-interpreted by means of simulation written in CSMP.

(2) The simulation programs give an extension to the logistic Lotka-Volterra equations. An abstract concept like the maximum population size has been replaced by physiologically relevant properties.

(3) Simulation shows that Gause's sampling technique is the main reason for the scatter in the case of the Paramecia rather than a stochastic birth-and-death process.

(4) The competition between two species has been explained with data and information derived from the monoculture only.

\section{REFERENCES}

Brennan, R. D., Wit, C. T. de, Williams, W. A. \& Quattrin, E. V. (1970). The utility of a digital simulation language for ecological modeling. Oecologia (Berl.), 4, 113-32.

Gause, G. F. (1934). The Struggle for Existence. Williams \& Wilkins, Baltimore.

Leslie, P. H. (1957). An analysis of the data for some experiments carried out by Gause with populations of the Protozoa, Paramecium aurelia and Paramecium caudatum. Biometrika, 44, 314-27.

Lotka, A. J. (1925). Elements of Physical Biology. Williams \& Wilkins, Baltimore.

Ross, G. J. S. (1972). Stochastic model fitting by evolutionary operation. Mathematical Models in Ecology (Ed. by J. N. R. Jeffers), pp. 297-308. Blackwell Scientific Publications, Oxford. 
Volterra, V. (1928). Variations and fluctuations of the number of individuals of animal species living together. Translation in: Animal Ecology (Ed. by R. N. Chapman), McGraw-Hill, New York (1934). Original in: J. Cons. perm. int. Explor. Mer, III, Vol 1 (1928).

Wit, C. T. de (1960). On competition. Versl. Landbouwk. Onderz., Wageningen, No. 66 (8), 1-82.

Wit, C. T. de \& Goudriaan, J. (1973). Simulation of Ecological Processes. Simulation Monographs, PUDOC, Wageningen.

(Received 26 June 1972) 



\section{BRITISH ECOLOGICAL SOCIETY PUBLICATIONS JOURNALS}

The Journal of Ecology

Edited by A. J Willis and P. J. GrubB. Published three times a year at $£ 5.25(\$ 17.00)$ per issue, postage extra: subscription price $£ 14.00$ ( $\$ 47.00)$ per annum, post free. $1973-$ Volume 61 ; all back volumes from Volume 31 currently available.

Index to Volumes $1-20(1913-32) £ 1.00(\$ 3.50)$

Index to Volumes 21-50 (1933-62) £2.25 (\$7.50)

Biological Flora of the British Isles 20p each (15p on standing order).

The Journal of Animal Ecology

Edited by H. N. SOUTHERN and L. R. TAYLOR. Published three times a year at $£ 5.25(\$ 17.00)$ per issue, postage extra; subscription price $£ 14.00(\$ 47.00)$ per annum, post free. 1973-Volume 42; all back volumes currently available.

Jubilee Symposium Supplement to the Journal of Ecology and the Journal of Animal Ecology. Edited by A. MACFADYEN and P. J. NewBould. 1964. 248 pages, 61 illustrations. $£ 2.50$ (\$8.50) postage $10 \mathrm{p}$ inland, $8 \mathrm{p}(\$ 0.25)$ abroad.

The Journal of Applied Ecology

Edited by T. H. CoAKer and H. W. Woolhouse. Published three times a year at $£ 5.25(\$ 17.00)$ per issue, postage extra; subscription price $£ 14.00(\$ 47.00)$ per annum, post free. 1973 -Volume 10; all back volumes currently available.

Pesticides in the Environment and their Effects on Wildlife A Supplement to Journal of Applied Ecology.

Edited by N. W. MOoRE. 1966. 324 pages, 35 illustrations. $£ 3.50(\$ 12.00)$.

\section{SYMPOSIA}

Biology in Pest and Disease Control

Edited by D. Price Jones and M. E. Solomon

Summer 1973.

The Measurement of Environmental Factors in Terrestrial Ecology

Edited by R. M. WADSWORTh. 1968 . $£ 2.75$

Quaternary Plant Ecology

Edited by H. J. Birks and R. G. West

Summer 1973.

About $£ 10.50$

The Teaching of Ecology

Edited by J. M. LAmbert. 1967.

$£ 2.87$

Mathematical Models in Ecology

Edited by J. N. R. JefFers. 1972.

$£ 5.50$

Scientific Management of Plant and Animal Communities for Conservation

Edited by E. A. G. Duffey. 1972. $£ 8.50$

Animal Populations in Relation to their Food

Resources

Edited by Adam Watson. 1970.

$£ 5.00$

Light as an Ecological Factor

Edited by R. BaInBridge, G. C. Evans and O. RACKHAM. 1966 (Reprinted 1968).

$£ 4.20$

Ecology and the Industrial Society Edited by G. T. Goodman, R. W. Edwards and J. M. LAMBert. $1965 . \quad$ Out of Print

Ecological Aspects of the Mineral Nutrition of Plants

Edited by I. H. RoBISON. 1969.

$£ 5.00$

Grazing in Terrestrial and Marine Environments

Edited by D. J. CrisP. 1964.

$£ 3.15$

\section{BLACKWELL SCIENTIFIC PUBLICATIONS OXFORD LONDON EDINBURGH MELBOURNE}

Printed in Great Britain by

Alden \& Mowbray Ltd at the Alden Press, Oxford 